\title{
High-Pressure Energy Dispersive X-ray Diffraction Investigation of Lithium-Manganese Spinel
}

\author{
Paweł Piszora \\ Laboratory of Magnetochemistry,Faculty of Chemistry, Adam Mickiewicz University, Poznań, \\ Grunwaldzka 6, PL-60780 Poznań, Poland
}

Keywords: $\mathrm{LiMn}_{2} \mathrm{O}_{4}$, phase transition, high-pressure structure.

\begin{abstract}
The formation of the tetragonal high-pressure structure, attributed to the Jahn-Teller distortion, was proved. The $c / a$ ratio shows a trend towards a stronger tetragonal distortion at high pressure. Rietveld refinement after the pressure relaxation shows the presence of tetragonal artefacts of high-pressure structure only for $\mathrm{LiMn}_{2} \mathrm{O}_{4}$ sample compressed in h-BN, whereas the sample compressed in silicone reveal the pure cubic phase. Confrontation with result of the first angledispersive diamond-anvil-cell experiment is presented.
\end{abstract}

\section{Introduction}

Lithium-manganese spinel oxide has been the subject of many previous investigations in different domains, however most studies have concentrated on the electrochemical character of the system, in view of its commercial application as a substitute for $\mathrm{LiCoO}_{2}$, due to being more environmentally friendly and costing less [1]. During the last 10 years several experimental studies on its high and low temperature phase transitions and also theoretical calculations have been carried out. The tetragonal structure of $\mathrm{Li}_{\mathrm{x}} \mathrm{Mn}_{3-\mathrm{x}} \mathrm{O}_{4}$ with $c / a>1$ has been observed by Tarascon at al. [2]. They have obtained the tetragonal sample with space group $I 4_{1} / a m d$. Subsequently, single crystal with composition $\mathrm{Li}_{0.91} \mathrm{Mn}_{0.09}\left[\mathrm{Mn}_{2}\right] \mathrm{O}_{4}$, obtained by a flux technique has been examined by Björk $a t$ al. [3]. It has been successfully indexed using tetragonal $I 4_{1} /$ amd space group and cell parameters $a=5.7502(9) \AA$ and $c=8.637(3) \AA$. In both papers, mentioned above, the body-centered standard space group has been applied. Nevertheless, the $c / a$ ratio is of 1.06 , after conversion to facecentered $F 4_{1} / d d m$ space group $\left(a_{\mathrm{F}}=\sqrt{2} a_{\mathrm{I}} ; c_{\mathrm{F}}=c_{\mathrm{I}}\right)$, more convenient for deformated spinels.

It is established that at ambient pressure the cubic-orthorhombic phase transition for pure $\mathrm{LiMn}_{2} \mathrm{O}_{4}$ occurs at about $280 \mathrm{~K}$. Lithium deficient $\mathrm{Li}_{1-\mathrm{x}} \mathrm{Mn}_{2+\mathrm{x}} \mathrm{O}_{4}$ samples obtained at $800^{\circ} \mathrm{C}$ and quenched rapidly in the solid $\mathrm{CO}_{2}$ exhibit two kinds of tetragonal structures, with $c / a>1$ and $c / a<1$ [4]. Recently, structural deformation in the series of the lithium deficient $\mathrm{Li}_{\mathrm{x}} \mathrm{Mn}_{3-\mathrm{x}} \mathrm{O}_{4}$ samples has been reported by author of this paper [5]. Samples with composition between $\mathrm{Li}_{0.85} \mathrm{Mn}_{2.15} \mathrm{O}_{4}$ and $\mathrm{Li}_{0.92} \mathrm{Mn}_{2.08} \mathrm{O}_{4}$ have revealed mainly the spinel phase with space group $F 4_{1} / d d m$ and $c / a 1.06$. As it has been proved, deficit of lithium on tetrahedral sites is filled in with $\mathrm{Mn}^{2+}$ ions, what leads to the reduction of average oxidation state of manganese in octahedral sites below +3.5 . In consequence, due to orientation of the tetragonally distorted octahedra along the $c$ axis, the cooperative JahnTeller effect is observed.

The effect of pressure on the structure of lithium-manganese spinel is as well of great interest. Previous experimental high-pressure investigations of the spinel structure oxides include studies of compressibility and phase transitions of many oxides, e.g. $\mathrm{ZnMn}_{2} \mathrm{O}_{4}, \mathrm{NiMn}_{2} \mathrm{O}_{4}, \mathrm{CuMn}_{2} \mathrm{O}_{4}$ [6], but only a few contradictive reports on the high-pressure investigations of lithium manganese spinel. Jak at al. [7,8] have not observed any significant structural changes after dynamic compaction, and successively relaxation from a pressure of $25 \mathrm{GPa}$. Nevertheless, the first in-situ high pressure experiment have show the existence of the new tetragonal phase indexed with $F 4_{1} / d d m$ space group [9]. The subsequent paper concerning investigation of $\mathrm{LiMn}_{2} \mathrm{O}_{4}$ at high pressure have suggested cubic orthorhombic phase transition, deduced from broadening of some cubic spinel lines [10]. In addition, those authors have observed $h k l$-dependent line broadening after the pressure relaxation, and from this fact they have concluded the presence of the quenched high-pressure non-cubic phase. 

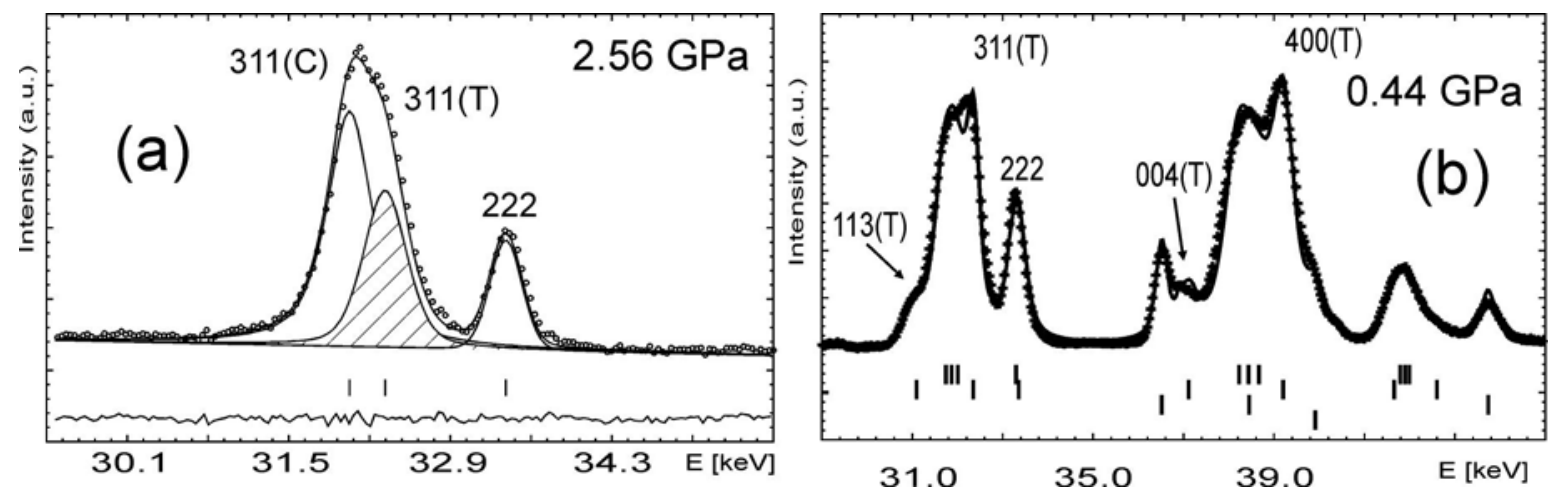

Fig. 1. Representative examples of peak fitting for $\mathrm{LiMn}_{2} \mathrm{O}_{4}$ in silicone, and Le Bail fitting of energy-dispersive pattern for the sample in h-BN as the pressure medium.

\section{Experimental}

$\mathrm{LiMn}_{2} \mathrm{O}_{4}$ sample was obtained by solid-state reaction of a- $\mathrm{Mn}_{2} \mathrm{O}_{3}$ precursor with $\mathrm{Li}_{2} \mathrm{CO}_{3}$ at $923 \mathrm{~K}$. The high-pressure powder diffraction experiments were performed using the energy dispersive X-ray diffraction method at a cubic anvil X-ray diffraction press, MAX80, installed at the DESY/HASYLAB F2.1 beamline. Samples were mounted in a boron nitride cylinder, inside a cylindrical graphite heater located in the middle of a boron-epoxy cube.

In the first pre-test experiment in a diamond anvil cell, the diamonds with $300 \mu \mathrm{m}$ culet diameter were used with spring steel gasket with $125 \mu \mathrm{m}$ hole. The data were collected on beamline I711 at the MAX-lab laboratory, Lund, Sweden. The pressure was measured using the ruby fluorescence method. Two dimensional diffraction patterns were collected using a Mar345 CCD detector system. Two-dimensional diffraction patterns were radially integrated using the program Fit2D [11] to obtain one-dimensional patterns.

\section{Results and discussion}

The formation of tetragonal structure, attributed to the Jahn-Teller distortion, was proved with Le Bail fitting [12], implemented in FullProf program [13]. For measurements in silicone gel the fitting of single reflections with WinPlotr program [14] were used to obtain the unit-cell volume change with pressure (Fig. 1).

The $c / a$ ratio shows a trend towards a stronger tetragonal distortion at high pressure (Fig. 2). This observation is contradictive to described for the $\mathrm{Mn}^{3+}$ silicate, where the stronger compression of the long bonds, relative to short bonds, leads to a smaller compressional overbonding, and acts against the Jahn-Teller distortion [15]. However, presented results coincide with the pressureinduced lowering of symmetry observed for any spinels with the Jahn-Teller $\mathrm{Mn}^{3+}$ ion [16].

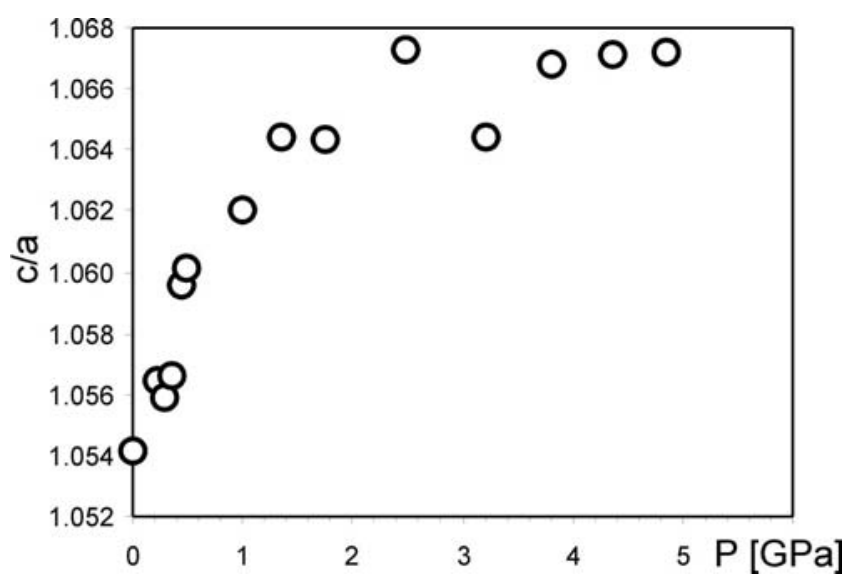

Fig. 2. The $c / a$ axial ratio of the high-pressure 'phase II' as a dependence of the applied pressure. 
Released $\mathrm{LiMn}_{2} \mathrm{O}_{4}$. After pressure relaxation the high-pressure phase of $\mathrm{NiMn}_{2} \mathrm{O}_{4}$ spinel has not reverted to the ambient-condition-stable cubic phase [17]. This fact has been interpreted as a consequence of hysteresis in the phase transformation. Considerable differences in the results of Rietveld refinement of released $\mathrm{LiMn}_{2} \mathrm{O}_{4}$ samples (Fig. 3) prove that the pressure medium is crucial for reversibility of the pressure phase transition. Sample compressed in silicone, revealed after relaxation the cubic $F d \overline{3} m$ structure. The noticeable splitting of cubic 311 and 400 reflections can
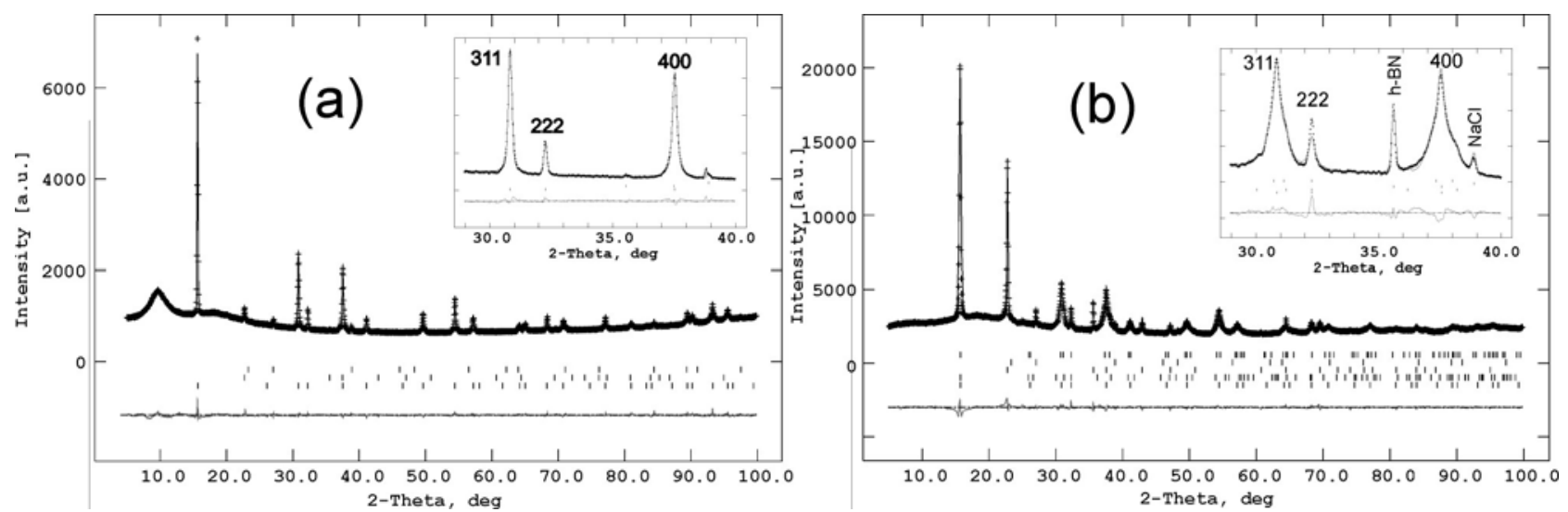

Fig. 3. Results of the Rietveld refinement of the synchrotron radiation powder diffraction patterns of $\mathrm{LiMn}_{2} \mathrm{O}_{4}$ formerly compressed in silicone (a) and hexagonal boron nitride (b), respectively.

be observed in pattern of released $\mathrm{LiMn}_{2} \mathrm{O}_{4}$ sample, earlier compressed in h-BN medium. It was fitted with mixture of the cubic and the tetragonal structures which could be assumed as artefacts of high-pressure structure. This observation should however be interpreted with caution, because of the non-sufficient angular resolution and difficulties in profile fitting due to microstructural effects. The measurements with higher resolution could give more structural details, nevertheless there are no evidence for relationship between the high-pressure structure, and that observed after relaxation.

$\mathrm{LiMn}_{2} \mathrm{O}_{4}$ in diamond-anvil cell. The angle-dispersive diamond anvil cell experiment seems to confirm the earlier observation of line splitting, suggesting the tetragonal phase with $F 4_{1} / d d m$ space group (Fig. 4). Unfortunately, this experiment did not allow to observe all diffraction lines of the tetragonal spinel phase, due to difficulties in extraction of ruby reflections and overlapping of lines

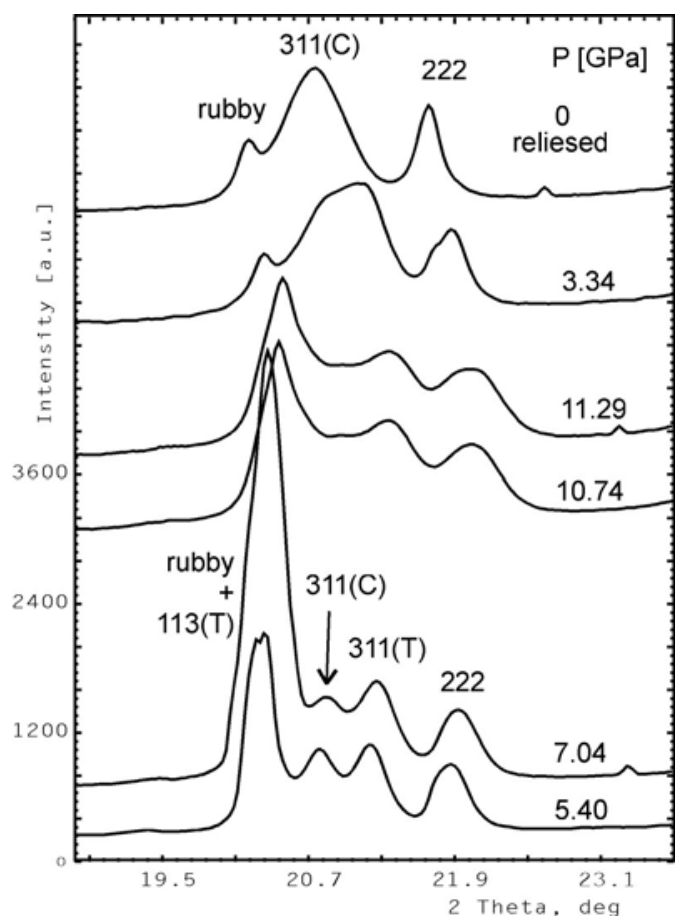

Fig. 4. Section of the integrated diffraction patterns of $\mathrm{LiMn}_{2} \mathrm{O}_{4}$ obtained in diamond-anvil cell. 
of the gasket. Waiting for solving technical problems in angle-dispersive mode, the energydispersive measurement is still the best technics of examination of the lithium-manganese spinels.

\section{Summary}

The symmetry lowering, arising in the compressed $\mathrm{LiMn}_{2} \mathrm{O}_{4}$ sample, can be attributed to a pressure-induced orientation of $\mathrm{Mn}^{3+} \mathrm{O}_{6}$ octahedra in the $c$-axis direction, as it is observed in lithium-deficient $\mathrm{Li}_{\mathrm{x}} \mathrm{Mn}_{3-\mathrm{x}} \mathrm{O}_{4}$ samples. Likewise, the $c / a$ axial ratio suggests the structure deformation analogous to that induced by the lithium deficit. Comparison of results of Rietveld refinement of a released $\mathrm{LiMn}_{2} \mathrm{O}_{4}$, formerly compressed in silicone and in h-BN, suggest significant importance of the pressure medium in preservation of structural distortion of the spinel lattice.

Acknowledgements. This work was supported by The Committee for Scientific Research (KBN) under grant No 128/E-336/SPB/DESY/KN/DWM/08/2005-2006. The author is grateful for the support from the European Community - Research Infrastructure Action under the FP6 "Structuring the European Research Area" Programme (through the Integrated Infrastructure Initiative "Integrating Activity on Synchrotron and Free Electron Laser Science".) by DESY/HASYLAB (Contract RII3-CT-2004-506008) and by MAX-Lab. The author would like to thank Dr. Yngve Cerenius and Dr. Stefan Carlson, both from MAX-lab (Lund), and to Dr. Christian Lathe from DESY/HASYLAB for assistance during the measurements.

\section{References}

[1] J.M. Tarascon and M. Armand: Nature Vol. 414 (2001), p. 359.

[2] J.M. Tarascon, W.R. McKinnon, F. Coowar, T.N. Bowmer, G. Amatucci and D. Guyomard: J. Electrochem. Soc. Vol. 141 (1994), p. 1421.

[3] H. Björk, H. Dabkowska, J.E.Greedan, T. Gustafsson and J.O.Thomas: Acta Cryst. C Vol. 57 (2001), p. 331.

[4] P. Piszora: J. Alloys Comp. Vol. 401 (2005), p. 34.

[5] P. Piszora: Chem. Mater. Vol. 19 (2006), (in press).

[6] L. Gerward, J.Z. Jiang, J. Staun Olsen, J.M. Recio and A. Waśkowska: J. Alloys Comp. Vol. 401 (2005), p. 11.

[7] M.J.G. Jak, E.M. Kelder, M. Stuivinga, and J. Schoonman: Solid State Ionics Vol. 86-88 (1996), p. 897.

[8] M.J.G. Jak, E.M. Kelder, J. Schoonman, V.N. Lashkov, A.V. Strikanov, A.A. Selezenev, and A.A. Potanin: J. Appl. Phys. Vol. 86 (1999), p. 5455.

[9] P. Piszora, W. Paszkowicz, W. Nowicki, R. Minikayev, and C. Lathe: HASYLAB Annual Report (2003), p. 309.

[10] A. Paolone, A. Sacchetti, P. Postorino, R. Cantelli, A. Congeduti, G. Rousse, and C. Masquelier: Solid State Ionics Vol. 176 (2005), p. 635.

[11] A.P. Hammersley: ESRF Internal Report, ESRF97HA02T, (1997).

[12] A. Le Bail, H. Duroy, and J.L. Fourquet: Mat. Res. Bull., Vol. 23 (1988), p 447.

[13] J. Rodriguez-Carvajal: FULLPROF version June 2005, ILL (unpublished).

[14] T. Roisnel, and J. Rodríguez-Carvajal: Mater. Sci. Forum, Vol. 321-324 (2000), p. 118.

[15] T. Armbruster, T. Kohler, E. Libowitzky, A. Friedrich, R. Miletich, M. Kunz, O. Medenbach and J. Gutzmer: Am. Mineral. Vol. 86 (2001), p. 147.

[16] A. Waśkowska, L. Gerward, J.S. Olsen, S. Steenstrup, and E. Talik: J. Phys. Condens. Matter Vol. 13 (2001), 2549.

[17] S. Åsbrink, A. Waśkowska, J.S. Olsen and L. Gerward: Phys Rev. B Vol. 57 (1997), 4972. 